\title{
Modelling Human Resource Systems in Support of Efficient Production
}

\author{
S.N. Khalil and R.H. Weston \\ Manufacturing System Integration (MSI) Research Institute, Wolfson School, \\ Loughborough University, Leicestershire United Kingdom, LE11 3TU
}

\begin{abstract}
Resource systems in manufacturing businesses need to be managed innovatively particularly when a range of products and services needs to be realized with minimum investment in resource systems. In this research, the primary form of active resource system, namely people, will be modelled with a view to facilitating efficient production. Humans play the key role in organising manufacturing businesses and contribute greatly towards competitive production performance. Enterprise Modelling is use to create static models that capture key organisational relationships in a manufacturing business and transformed into equivalent structures within Simulation Models that enable dynamic analysis. This provides both graphical and computer executable models of people and facilitates quantitative analysis and comparison of different human system configurations that suit various manufacturing workplaces. The paper illustrates the application of the modelling approach and observes advantages gained through using coherent sets of enterprise and simulation models.
\end{abstract}

Keywords: Human system, manufacturing enterprise, simulation modelling, process-oriented enterprise modelling.

\section{Introduction}

To be able to design an efficient production system, designers and managers of Manufacturing Enterprises (MEs) need to be able to reason about the assignment of persons to production activities such that key performance measures (like lead-time, quality and cost targets) are met. Typically in industry this is done implicitly with regard to 'abilities' (of alternative candidate resources (including people, semi- automated machines and supporting IT systems) to fulfill a given set of product realising roles with respect to specific case performance measures. When assigning production operatives to roles, production system designers and managers use their experience and knowledge about abilities of the people involved and makes judgments about their individual and collective abilities to accomplish predicted patterns of upcoming work. However often production systems, and/or the products they make, can be complex. Indeed when production systems need to share human and technical (machine and IT) resources amongst a changing mix of multiple value streams, frequently designers/managers may make poor people and machine assignment decisions, which will lead to poor attainment of production targets that causally may result in poor competitive performance of the manufacturing business as a whole. In such case 
managers/designers can gain significant competitive advantage from the ongoing reuse of an effective decision-support tool which will help them make better informed decisions about how and when to allocated a finite production resource in response to any given customer induced dynamic.

This paper researches a new means satisfying this requirement for decision support. The approach taken is to deploy a synergistic combination of:

- Enterprise Modelling, which explicitly describes organisational structures related to a particular parent manufacturing business; so that process-oriented structural relationships inter-connecting production systems are uniquely defined, and

- Simulation Modelling, which is capable of simulating time-based behaviours of alternative production system configurations of people and technical resource; thereby potentially enabling production system designers and managers, in a virtual environment, to experiment within systematic changes to organisational structures and production system parameters so as to predict changes in production system behaviours and therefore performance outcomes in respect of new work scenarios.

The modelling approach taken has potential within dynamic production systems environments to much improve the deployment of a constrained set of people and machine resources and further by predicting change outcomes prior to making physical production system change the costs and benefits of changes trial can be assessed, thereby protecting risk associated with production system investments and potentially extending the economic life-time of production systems. This paper describes how an enterprise model can be used to capture a big picture of the organisational structures of a case study Manufacturing Enterprise (ME). The approach being researched has potential to form the basis of a decision support tool, which can repetitively, quantitatively and explicitly inform production system designers/manager about the suitability of different assignments of human and technical resources, given a set of changing work. Therefore this paper is design to aid the efficient deployment of human systems in MEs by:

- $\quad$ Proposing a human system modelling technique which can be used on an ongoing and systematic basis to model, understand and engineer human systems that satisfy dynamic production system requirements;

- $\quad$ Analyzing key aspects of human system dynamics by developing static and dynamic models of human systems.

The paper will discuss the background of the research work. It will also explain how Enterprise (static) and Simulation (dynamic) modelling can be used in a complementary fashion to visualise and computer executes alternative possible human system deployments.

\section{Manufacturing Complexity}

Over many years great interest has been placed in managing complexity in manufacturing organizations. Siemieniuch and Sinclair[1] defined manufacturing complexity from an organisational perspective. They state that if interactions between entities of 
an organisation give rise to unpredictable organisational performance then that organisation is complex. Common entities in manufacturing organisations comprise human resources, electromechanical and IT machines which typically are organised to realise customer related processes and infrastructure services [2].

When seeking to manage complexity, various approaches and manufacturing paradigms have been introduced. For example the Group Technology (GT) has widely been used in industry to improve manufacturing processes by means of grouping and coding. GT types of activities are also referred as classification and clustering: of parts that are similar in nature; or parts that require operations of similar machinery and processes[3]. Advantages of deploying GT philosophy are (1) to simplify the flow of parts and tools, (2) to reduce set-up times, (3) reduce throughput time, (4) reduce work-in-process inventory and (5) maximize design and manufacturing efficiencies. However according to name Kusiak and Heragu, the use of a clustering method may not be efficient because of constraints from needed information flows in the processes; such as if data related to material, geometry and tolerances is not available.

The average lifetime of products has shortened dramatically in the last 10 years. Product variations have increased and this requires MEs to be more flexible and responsive towards their customer demands. But current trends in global demand mean that more products are now produced in low quantities, with high variety amongst demand patterns. This is because increasingly products are highly customised and/or their underlying technologies and components are subject to increased rates of change. Richards states that being responsive and agile is as being able to respond to sophisticated customer requirements when subject to persistently changing competitive processes and success factors [4].

\section{Trends and Needs to Model Human Systems}

\subsection{Currents Trends in Manufacturing Industry}

The new millennium has seen rapid change in many aspects and segments of manufacturing industries. Some factors contributing towards this change are:

- Intense global competition.

- Mass markets fragmenting into niche markets.

- Customers expecting low volume, high quality, custom products.

- Seemingly ever shortening product life-cycles, development times, and production lead times are required.

- Customers want to be uniquely treated, i.e. as individuals

In an attempt to address these factors, organisations need much more frequently to be re-configured. This for example may require some form of change in human oriented organisational structures. Manufacturing organisations, basically comprise human resources, and electromechanical and IT machines; and in general systematic use of these 'active' resources (i.e. resources that can perform required ME actions) are commonly organised so that they systematically achieve value adding processes and related infrastructure support and engineering developmental activities (Pandya et al 1997). Earlier in this paper, the literature focussed on the paradigms and approaches 
that industry is adopting to improve manufacturing processes. However these paradigms were mainly discussed in respect of their mechanistic aspects rather than their behavioural aspects and how such behaviours can impact on MEs. However the functional aspects of human-related processes still requires further study. As the prime ME resource, there is a great need for humans to know about the 'how', 'what' and 'do' (BS ISO 14258:1998) activities in a given ME and the related roles that they and other humans can play with respect to manufacturing activities. The literature reviewed in this paper illustrates that there is a gap in quantitative understandings about potential roles of technical (electromechanical and IT) resources and related roles that human resources can play with respect to engineering, infrastructural and direct value adding manufacturing activities.

\subsection{Needs to Model Human System}

As yet there is no widely accepted (by industry or academia) systematic way to qualitatively and quantitatively model ME process oriented roles; and how such roles may function over time under differing production demand patterns should they be resourced by humans and machines or by humans or machines. In general it is observed that addressing this deficiency requires effective models of relationships between human/human and human/machine, which may be uncertain in nature. Therefore it is observed that great advantage could be gained by having a 'simulation tool' to model alternative combinations of resource entities; so as to inform 1) managerial aspects of human/machine resource allocation, by providing support for planning decisions and determining suitable resource system reconfigurations based on predictions about organisational behaviours and 2) design aspects of production systems, associated with mid to long term investment decision making.

\section{Human System Modelling in Manufacturing Enterprise}

Humans are unique and their behaviour can be difficult to predict. Thus it may be almost impossible to model the unconstrained human due to these complexities. On the other hand, potentially human systems can be modelled from more constrained viewpoints. Example viewpoints include: psychology, physiology, culture, structure, dynamic, roles and attribute. Researchers in the MSI (Manufacturing System Integration Institute) have focused their human systems modelling efforts on modelling people roles (i.e. organised sets of activities that people perform) and to consider how the abilities and behaviours of potential human role holders impact on achieving production targets. The approach being taken is therefore based in process oriented modelling. One thread of top-down modelling followed is centered on ME process decomposition into hierarchy/levels for action taking and information sharing. Here processes are explicitly and graphically modelled as related sets of enterprise activities (EAs) which identify possible sets of explicitly defined roles that can be resourced by alternate groups of people competent to perform the role sets defined. Here role sets essentially specify a process oriented organisational structure. This is illustrated by figure 1 . The activities are defined in terms of unitary functional operations (FO) that require unitary functional entities (FE) which can be possessed by human and/or machine holders of 
those roles. In a second thread of modelling candidate human (and machine) holders of roles are described in a tabular fashion, in terms of the competencies they can bring to bear on defined role sets (which can be matched to FE) and likely behavioural performances they can achieve (which can be linked to FO sequence models). A third thread of modelling is centered on simulation of alternative candidate configurations of roles and role holders, which provides a tool for comparing throughputs, utilisation, cost and value generation abilities of those alternative configurations.

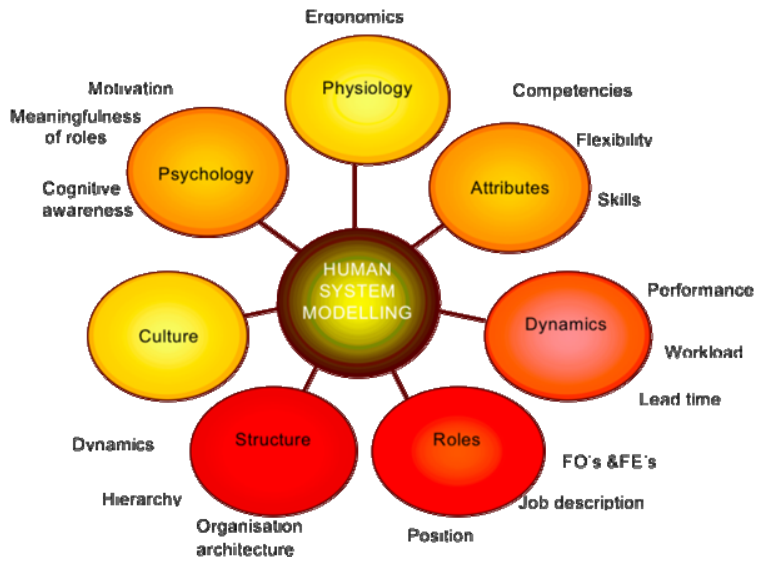

Fig. 1. Human system entities

\section{Modelling Concepts and Research Methods}

MSI researchers have been using enterprise engineering and simulation modelling technique to aid decision-making. Enterprise modelling is used primarily to (1) externalizing enterprise knowledge about case study MEs, and can add value to the enterprise by enabling knowledge sharing and (2) to provide a process-oriented decomposition mechanism, so that high levels of complexity can be handled and it becomes possible to break down the barriers in organization that hinder productivity by synergizing the enterprise to achieved better understandings about how business goals can be achieved in an efficient and productive way[5].

The enterprise modelling (EM) technique used in this study is known as CIMOSA. CIMOSA is an acronym derived from CIM Open System Architecture and this acronym was introduced by the AMICE consortium. In CIMOSA the user representation and system representation, and related function information and control perspectives are decomposed. The associated decomposition and isolation of different modelling concepts and viewpoints enables an organization to be represented in a flexible fashion, so as to realise changing requirements for functional and facilities integration. Generic graphical representation of CIMOSA models is illustrated in figure 2. CIMOSA modelling enables ME decomposition into the following: 
- Domains (DM)

- Domain Processes (DP)

- Business Processes (BP) and
- Enterprise Activities (EA)

- Functional Operators (FO)

- Functional Entities (FE)

This case study modelling is supported by simulation models (dynamic models) that are in part derived from (and hence are consistent with) selected segments of the CIMOSA static model. CIMOSA graphical models are static in the sense that they only encode relatively enduring properties of MEs and cannot be computer executed to show ME behaviours over time. The simulation models are capable of modeling queues, stochastic events, product flows, process routes, resource utilisation, breakdowns and absence, exception flows and etc. Enterprise and simulation modeling (SM) techniques can mutually support analysis of human system roles in a manufacturing enterprise.

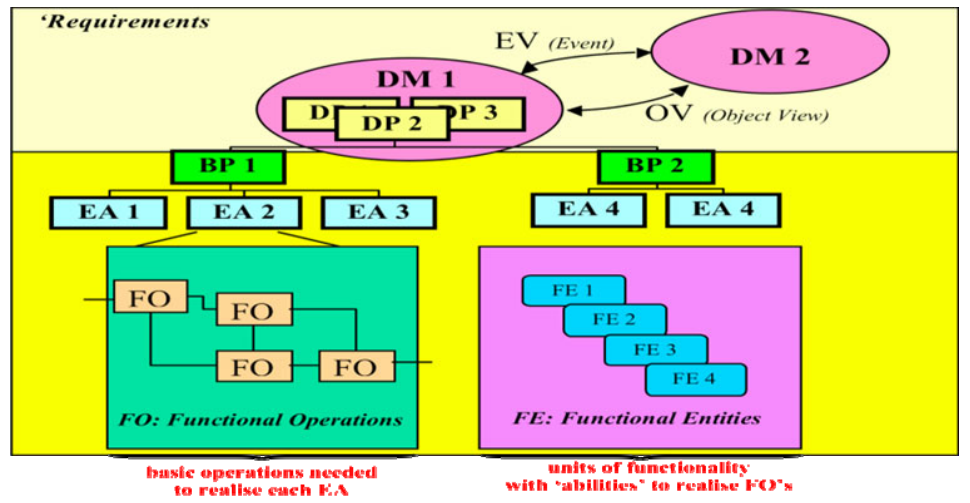

Design Specificarion"

Fig. 2. CIMOSA static model

\section{Research Domain, Integration of Enterprise and SM}

Chosen case study company is an air conditioning company, LS a privately owned Chinese manufacturing company located in Shun De in the Guangdong province of South China. Since 1988 when it came privately owned, it has expanded its manufacturing and supply scope and is currently specialised in the 'engineering to order' of customised industrialised air conditioners. Their unique expertise is demonstrated in their competence in meeting varying customer needs. On the average LS employs about 1000 staff of which 60 are mainstream Engineers. Most of the staff is young graduates from universities and colleges and hence the company exhibits high level of exuberance in terms of its personnel. The assembly shop 2 has been chosen as the case study for this research. The shop floor assembly held in five stages:1)general assembly, 2)pipes and tubing and hydraulic tests, 3)electronic control installation and side panels assembly, 4) testing and lastly 5)packaging. Each stages of the assembly work is supported by 66 staff that is grouped into 5 sets of different teams. Each team are trained and specialised in each stages. The process that took place during the assembly is described in CIMOSA enterprise activity diagram BP 6.3.1 as referred in figure 3. 
The simulation modelling (SM) of these workstations is as described in figure 5 . The simulation modelling is held using Teknomatix Plant Simulation Modelling software tool. The software is a custom made tool for users that specifically designed to model the performance of a manufacturing plant. The SM is capable of modelling human resources hierarchically. The broker or acting as supervisor worked by assigning workers in the workpool to perform activities/processes at the workstations. Workstations are the places where worker is assigned to do their work assigned at each stage and the performance is affected by i.e. workers efficiency, dynamic input etc.

In this case the efficiency of the workers are set to $60 \%$ efficient in the first experiment and the other is set $80 \%$ efficient in the second experiment. Thus is done to compare the performance of the production line when workers efficiency is changed.

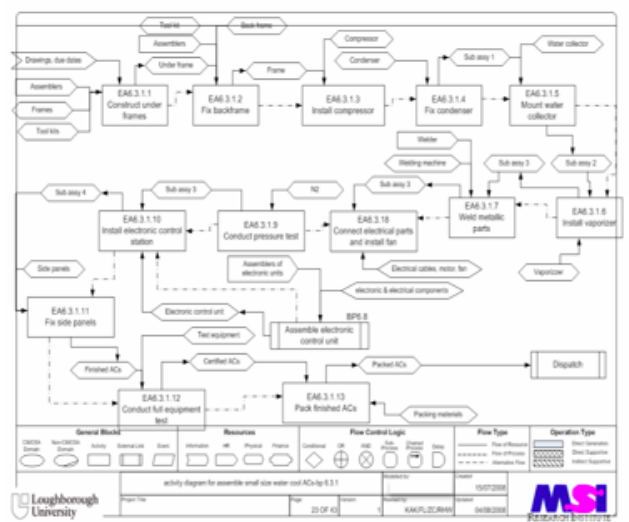

Fig. 3. Activity Diagram-Assembly shop 2

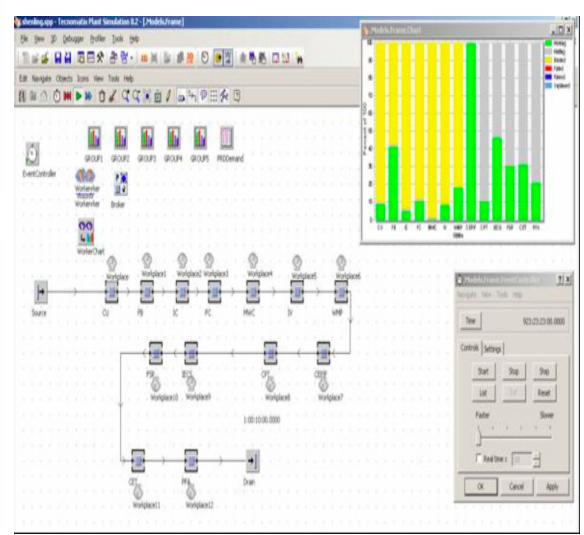

Fig. 4. SM of AC assembly

The chart of the overall performance of the work centers is shown in figure 4 . The column in the chart shows the pattern of working and blocked processes taking place during the simulation is done. The simulation throughput results showed that there is a $30 \%$ increase of production throughput i.e. from $1 \mathrm{AC}$ unit per day to $1.3 \mathrm{AC}$ units per day when the efficiency of workers increased from $60 \%$ to $80 \%$. When the number of workers in Team 1 is reduced by half, the simulation results showed that the throughput remained to 1 per day when the resource efficiency is set to $80 \%$. The results showed there is potential for the production system design to reduce the amount of workers when the workers are working at higher efficiency.

\section{Further Work in Human System Modelling in Manufacturing Enterprise}

Current modelling is based on the 'as is' model, i.e. the current process carried out in the particular bearing manufacturing system. Improvements that will be performed in later stages include: 
1) Improving matching roles to role holders in Static Model for modelling and allocating the right worker for the right processes according to different competency levels of workers.

2) Enhancing the current team with abilities to perform multiple processes and able to work in cross-team assembly work. This will improve the throughput of the process line due to increase of shared-knowledge and information flow in the assembly line.

\section{Summary and Conclusions}

This approach has developed new understandings about different types of model that can be used to characterize human system dynamics. This characterization is based on a job order specification at which influenced by multiple variances i.e. product specification variances. At this stage of experimentation the author has not yet been able to find out whether these models can usefully predict the impact of the overall performance of manufacturing enterprise due to product variance. But early results are encouraging.

\section{Acknowledgments}

The principal researcher is currently a $\mathrm{PhD}$ researcher in Loughborough University and sponsored by the Malaysian Ministry of Higher Education/ Universiti Teknikal Malaysia Melaka.

\section{References}

1. Siemieniuch, C.E., Sinclair, M.A.: On complexity, process ownership and organisational learning in manufacturing organisations, from an ergonomics perspective. Applied Ergonomics 33(5), 449-462 (2002)

2. Pandya, K.V., et al.: Towards the manufacturing enterprises of the future. International Journal of Operations \& Production Management 17(5), 502-521 (1997)

3. Kusiak, A., Heragu, S.S.: Group technology. Computers in Industry 9(2), 83-91 (1987)

4. Richards, C.: Agile manufacturing beyond lean. Production and Inventory Management Journal, 60-64 (1996)

5. Vernadat, F.B.: Enterprise Modelling and Integration. Chapman and Hall, London (1996) 